\title{
Influência dos métodos contraceptivos hormonais e dispositivo intrauterino na prevenção de infecções sexualmente transmissíveis em mulheres jovens
}

\author{
Influence of hormonal contraceptive methods and intrauterine devices on the prevalence of \\ transmitted infections in young women \\ Influencia de los métodos anticonceptivos hormonales y dispositivos intrauterinos en la prevalencia \\ de infecciones de transmisión sexual en mujeres jóvenes
}

Recebido: 23/07/2021 | Revisado: 30/07/2021 | Aceito: 01/08/2021 | Publicado: 07/08/2021

\author{
Camila Maria Pinazo Jacques \\ ORCID: https://orcid.org/0000-0002-5797-0257 \\ Centro Universitário da Fundação Assis Gurgacz, Brasil \\ E-mail: camilapjacques@gmail.com \\ Winny Hirome Takahashi Yonegura \\ ORCID: https://orcid.org/0000-0001-9968-4235 \\ Centro Universitário da Fundação Assis Gurgacz, Brasil \\ E-mail:wy1980@hotmail.com \\ Larissa Kerr de Araújo Sodré \\ ORCID: https://orcid.org/0000-0003-3877-5218 \\ Centro Universitário da Fundação Assis Gurgacz, Brasil \\ E-mail: larissa_kerr@hotmail.com
}

\begin{abstract}
Resumo
O objetivo deste estudo é analisar se o uso de métodos contraceptivos hormonais e Dispositivo Intra-Uterino (DIU) pode influenciar na utilização de métodos contraceptivos de barreira, avaliando se existe associação com aumento na prevalência de Infecções Sexualmente Transmissíveis (ISTs) em mulheres jovens (de 20 a 30 anos). Foram analisados prontuários no período de 2018 a 2019 do Centro Especializado em Doenças Infecto-parasitárias (CEDIP) do município de Cascavel-PR, de mulheres diagnosticadas com infecções sexualmente transmissíveis que se encaixam na faixa etária determinada. A maioria das mulheres diagnosticadas com IST utilizam como método contraceptivo anticoncepcional ou dispositivo intrauterino (58\%). Destas mulheres, apenas $45 \%$ fazem uso concomitante de algum método de barreira e destas, $59 \%$ o utilizam de maneira irregular. Dessa forma, constatou-se que $81 \%$ das mulheres com ISTs não utilizam preservativo de barreira ou o utilizam de maneira incorreta. Através de análises inferenciais dos dados obtidos, foi possível estabelecer uma hipótese com valor estatisticamente significativo, ou seja, valor $\mathrm{p}<0,05$ de que mulheres que utilizam contraceptivo de barreira contraem menos ISTs que mulheres que escolhem outros métodos contraceptivos, como é o caso dos anticoncepcionais hormonais e do DIU. Esse resultado respalda a hipótese do projeto que somente o uso de contraceptivos hormonais ou DIU não protege contra a disseminação de ISTs.
\end{abstract}

Palavras-chave: Doenças Sexualmente Transmissíveis; Anticoncepcionais orais hormonais; Dispositivo intrauterino; Prevenção de doenças.

\begin{abstract}
The objective of this study is to analyze how the use of hormonal contraceptive methods and intrauterine devices (IUD) can influence the use of barrier contraceptive methods, assessing whether there is an association with increased prevalence of sexually transmitted infections (STIs) in young women (aged 20 to 30 years). Were analyzed medical records of the years 2018 and 2019 of the Specialized Center for Infectious and Parasitic Diseases (CEDIP) in CascavelPR, of women diagnosed with sexually transmitted infections that fit the specific age range. Most women diagnosed with STI use hormonal contraceptive or intrauterine device (58\%). Of these women, only $45 \%$ make concomitant use of some barrier method and of these $59 \%$ use it irregularly. Thus, it was found that $81 \%$ of women with STIs do not use barrier condoms or use them incorrectly. Through inferential analyzes of the data obtained, it was possible to establish a hypothesis with a statistically significant value, that is, $\mathrm{p}<0.05$, that women using barrier contraceptives contract less STIs than women who choose other contraceptive methods, such as hormonal contraceptives and IUD. This result supports the project's hypothesis that only the use of hormonal contraceptives or IUD does not protect against the spread of STIs.
\end{abstract}

Keywords: Sexually Transmitted Diseases; Oral hormonal contraceptive; Intrauterine device; Disease prevention. 


\section{Resumen}

El propósito de este trabajo fue analizar cómo el uso de métodos anticonceptivos hormonales y dispositivos intrauterinos (DIU) puede influir en la utilización de métodos anticonceptivos de barrera, evaluando si existe una asociación con aumento en la prevalencia de infecciones de transmisión sexual (ITS) en mujeres jóvenes (de 20 a 30 años).Se estudio las historias clínicas registradas entre los años 2018 y 2019 en el Centro Especializado en Enfermedades Infecciosas y Parasitarias (CEDIP) de Cascavel-PR, de mujeres diagnosticadas con infecciones de transmisión sexual que se ajustan al rango de edad específico. La mayoría de las mujeres diagnosticadas con ITS utilizan como método anticonceptivo o dispositivo intrauterino (58\%). De estas mujeres, solo $45 \%$ hace uso concomitante de algún método de barrera y de estas, 59\% lo usa de manera irregular. Así, se encontró que $81 \%$ de las mujeres con ITS no usan condones de barrera o los usan de manera incorrecta. A través del análisis inferencial de los datos obtenidos, fue posible establecer una hipótesis con un valor estadísticamente significativo $(\mathrm{p}<0.05)$ que las mujeres que usan anticonceptivos de barrera contraen menos ITS que las mujeres que optan por otros métodos anticonceptivos, como los anticonceptivos hormonales o el DIU. Este resultado apoya la hipótesis del proyecto de que solo el uso de anticonceptivos hormonales o DIU no protege contra la propagación de las ITS.

Palabras clave: Enfermedades de Transmisión Sexual; Anticonceptivos hormonales orales; Dispositivos intrauterinos; Prevención de enfermedades.

\section{Introdução}

A incorporação da pílula anticoncepcional no mercado durante a década de 70 foi um passo a mais na ruptura dos padrões tradicionais de reprodução e na nova dinâmica populacional no país, proporcionando às mulheres, que estavam cada vez mais aumentando sua participação no mercado de trabalho, um maior controle da sua fertilidade e do seu corpo (Vieira et al, 2002). Porém, embora tenha sido um grande avanço no planejamento familiar e ganhado alta popularidade com o passar dos anos, há também um lado negativo: pode haver displicência com o uso de preservativos, gerando uma maior disseminação de infecções sexualmente transmissíveis (IST).

Um dos principais métodos contraceptivos utilizados são os hormonais, representados pelo anticoncepcional oral, injetável e sistema uterino liberador de levonorgestrel, que são contraceptivos reversíveis que atuam interrompendo a ovolução e/ou fecundação e, por consequência, a gravidez (Grandi et al, 2019). A eficácia desses métodos, medida através do índice de Pearl (número de gestação em 100 mulheres que utilizaram o método durante um ano) é superior ao preservativo de barreira masculino. Por exemplo, o índice de Pearl varia de 0 a 1.26 no uso perfeito e 0 a 2.18 em uso típico, considerando pílula contraceptiva oral combinada (Mansour et al, 2010). Contudo, embora estes métodos sejam efetivos em evitar gravidez eles não evitam transmissão de ISTs e em geral podem apresentar alguns efeitos colaterais e contraindicações clínicas (Serfaty, 2019; Baeten et al, 2001).

O Dispositivo Intra-Uterino (DIU) de cobre é um dos métodos contraceptivos mais efetivos, apresentando índice de Pearl de 0,52 e não é afetado pela quantidade de massa corporal. Embora apresente maior efetividade que métodos de barreira ao evitar gravidez indesejada este método, assim como métodos hormonais, não evitam transmissão de ISTs (Centers For Disease Control and Prevention, 2015; Apter, 2017; Mansour, 2010; Baeten et al, 2001).

Ao contrário dos métodos hormonais e DIU, os métodos contraceptivos de barreira, como o condom masculino e feminino atuam prevenindo ISTs se usados corretamente (Holmes et al, 2004; Decker, 2016). Contudo, dados da literatura afirmam que a eficácia contraceptiva é inferior aos métodos hormonais (Mansour et al, 2010). Usuários que apresentam alergia ao látex podem utilizar de forma alternativa condoms fabricados com outros materiais como poliuretano (Colquit e Martin, 2017; Gallo et al, 2013).

Dessa forma, usuários que utilizam métodos contraceptivos de barreira corretamente apresentam maior proteção para ISTs, que são infecções causadas por uma diversidade de agentes patológicos, majoritariamente transmitidas através de relações sexuais (vaginal, anal ou oral) sem a utilização de preservativos, embora possam ser disseminadas por contágio sanguíneo ou de mãe para filho na gravidez e amamentação (Brasil, 2015). 
Atualmente são estimados 376 milhões de novos casos por ano de ISTs curáveis (sífilis, clamídia, gonorreia e tricomoníase), além de outros tantos milhões de ISTs não curáveis, incluindo o herpes genital, infecções pelo Papilomavírus Humano (HPV), hepatite B e infecção pelo HIV. A abrangência da transmissão dessas infecções causa grandes preocupações para a sociedade, tendo em vista as consequências causadas por essas condições, tais como: infertilidade, gravidez ectópica, resistência dos microrganismos aos tratamentos e aumento do risco de infecção por HIV (Organização Mundial da Saúde, 2019).

Algumas ISTs como sífilis apresentam incidências crescentes em países como Estados Unidos e Brasil (Mccormack e Koons, 2019; Dos Santos et al, 2020). Causada por uma espiroqueta denominada Treponema pallidum, é conhecida por ser "a grande imitadora" pela grande quantidade de apresentações e sintomas causados pela infecção, como condiloma lata, linfadenopatia e lesões mucocutâneas podendo ficar latente por anos até evoluir para sífilis terciária (Ministério da Saúde, 2020; Centers For Disease Control and Prevention, 2018; Mccormack e Koons, 2019).

As cervicites são frequentemente assintomáticas. Os agentes etiológicos principais são Chlamydia trachomatis e Neisseria gonorrhoeae. No Brasil existe grande dificuldade em dados de prevalência já que a pesquisa de clamídia e gonococo não está disponível na rede pública, apesar de ser sugerida em várias situações pelo próprio Ministério da Saúde (Ministério da Saúde, 2020). A clamídia é a IST bacteriana com maior frequência nos Estados Unidos, com 528,8 casos a cada 100.000 habitantes (Centers For Disease Control and Prevention, 2018). Também apresenta grande variedade de apresentações e sintomas como dor pélvica e retal, secreção vaginal, disúria e pode evoluir para infertilidade, gravidez ectópica e doença inflamatória pélvica (Mishori et al, 2012; Park et al, 2017; Den Heijer et al, 2019).

É recomendado pelo CDC americano triagem anual de mulheres sexualmente ativas até 25 anos e todos homens que têm relações sexuais com homens (Centers For Disease Control and Prevention, 2015). Da mesma forma, a gonorreia apresenta taxa anual de 171,9 casos por 100.000 habitantes, sendo a segunda IST bacteriana mais comum (Mccormack e Koons, 2019; Centers For Disease Control and Prevention, 2018). Apresenta recomendações de triagem semelhantes as da clamídia, podendo manifestar-se com secreção/corrimento vaginal ou peniano, vaginose, dor pélvica, articular ou retal (Centers For Disease Control and Prevention, 2018; Mitchell e Prabku, 2013; Chan et al, 2016).

Herpes simples pode ser causado pelo vírus do tipo 1 (também conhecido como herpes labial, embora também possa acometer a região genital) e tipo 2 (conhecido como herpes genital, embora possa acometer a região facial) (Fuchs e Brockmeyer, 2014). Os sinais e sintomas característicos são vesículas dolorosas em fundo eritematoso (Fuchs e Brockmeyer, 2014; Baldivia e Graudenz, 2015)

A infecção por tricomonas pode apresentar-se como uretrite, prostatite ou epididimite em homens, e na mulher, tipicamente, com uma secreção vaginal, geralmente verde-amarelado com odor fétido (Wagenlehner et al, 2016). Embora possa ser assintomática ou ter poucos sintomas em 70 a $85 \%$ das pessoas, a infecção por tricomonas vaginalis pode elevar o risco de adquirir HIV por um fator de 2 a 3 (Kissinger e Adamski, 2013; Wagenlehner et al, 2016).

O HPV pode induzir uma grande variedade de lesões cutaneomucosas, desde verrugas anogenitais até câncer de colo uterino (Crosbie et al, 2013). Além do colo uterino, o HPV também pode causar câncer na vulva, vagina, ânus, pênis e orofaringe (De Martel et al, 2012). Sua transmissão pode ocorrer com qualquer tipo de atividade sexual e menos frequentemente de mãe para filho durante o parto. Embora exista vacinação profilática efetiva, a prevalência do HPV é elevada (10,4\% das mulheres) (Sanjosé et al, 2007) e, dessa forma é esperado que o efeito da vacinação leve mais que 20 anos para ter efeito no câncer cervical (Crosbie et al, 2013; Sanjosés et al, 2007).

O Vírus da Imunodeficiência Humana (HIV) é transmitido majoritariamente por contato sexual. Em estudos na Alemanha, cerca de $90 \%$ dos pacientes soropositivos adquiriram a infecção por meio de contato sexual (Wagenlehner et al, 2016). A presença de outras ISTs também amplia a transmissão do HIV (Galvin e Cohen, 2004). Por apresentar período de latência clínica prolongado o diagnóstico tardio é um problema sério e comum, pois pacientes com estágio avançado de 
imunodeficiência ( $<200 \mathrm{CD} 4 / \mu \mathrm{L}$ ) apresentam piora na evolução clínica além de elevar o risco de transmissão (Wagenlehner et al, 2016; Rouzine, 2020; Crosbie et al, 2013).

Apesar do avanço da medicina nas alternativas de prevenir e tratar as ISTs, o processo ainda pode ser demorado e danoso, além de muitas dessas infecções não terem cura. Por isso, uma medida de muita importância é a disseminação de informação e o ensino da educação sexual, já que apesar de todas as alternativas de métodos contraceptivos serem eficientes contra a fecundação, apenas os preservativos masculino e feminino são capazes de impedir a transmissão de infecções sexuais (Petry et al., 2019).

Grupos especiais de mulheres, como adolescentes, apresentam alta taxa de gravidez indesejada e ISTs. Dessa forma, é importante oferecer educação contraceptiva para elas tomarem decisões informadas e livres de coerção considerando os diversos métodos disponibilizados (Raidoo e Kaneshiro, 2017). Principalmente neste grupo de pacientes é recomendado a dupla proteção, com a utilização de dois métodos contraceptivos, sendo um hormonal e outro de barreira, para maximizar proteção contra gravidez indesejada associada a proteção com ISTs (Apter, 2017).

Dessa forma, este estudo buscou avaliar a influência do uso de métodos contraceptivos hormonais no aumento da prevalência das IST, verificando o uso de condom/preservativo de barreira associado ou não ao uso dos métodos hormonais.

\section{Metodologia}

Trata-se de um estudo transversal, quantitativo e observacional, com dados advindos do prontuário de mulheres na faixa etária entre 20 e 30 anos que foram atendidas no Ambulatório Municipal de ISTs no Centro Especializado em Doenças InfectoParasitárias (CEDIP) na cidade de Cascavel-PR e diagnosticadas com alguma IST durante os anos de 2018 e 2019. A pesquisa foi aprovada pelo Comitê de Ética em Pesquisa em Seres Humanos do Centro Universitário da Fundação Assis Gurgacz com número CAEE 38669220.0.0000.5219. Como base metodológica foi utilizado o trabalho de Pereira et al (2018).

Os dados de diagnóstico e método contraceptivo utilizado serão a base para comprovar se há ou não uma real relação entre a utilização de contraceptivos hormonais e o aumento da transmissão de ISTs e, dessa forma, estabelecer uma possível contribuição para o desenvolvimento de melhores políticas de promoção de saúde e prevenção das infecções sexualmente transmissíveis.

Os testes estatísticos de associação foram realizados no software RStudio na versão 1.4.1103, utilizando os testes de Fisher e Qui-Quadrado para comparação de frequências entre os grupos. Para a avaliação de diferenças de medianas entre os grupos utilizou-se o teste de Mann-Whitney. Foram consideradas as hipóteses alternativas estatisticamente relevantes aquelas com valor de p inferior ao nível de significância $(\alpha)$ de 0,05 .

\section{Resultados e Discussão}

Foram coletados, através de prontuários, informações referentes às 127 pacientes atendidas em um centro de atenção secundária (Tabela 1), de forma a realizar uma análise de possíveis associações que justifiquem o aumento do número de ISTs, especialmente nas populações mais jovens (Ministério da Saúde, 2020).

Destaca-se que 75 mulheres (58\%) diagnosticadas com IST utilizam anticoncepcional ou dispositivo intrauterino como método contraceptivo. Destas, 41 (55\%) usam somente métodos hormonais ou DIU como método contraceptivo, e apenas 34 (45\%) fazem uso concomitante de método de barreira. Ainda, dentre essas pacientes que fazem uso dos métodos hormonais associados ao preservativo, a maioria (59\%) relatou fazer uso deste último de maneira irregular. Logo, somando-se as pacientes que não fazem uso de preservativo com as que o fazem de maneira incorreta, tem-se $81 \%$ das pacientes (Tabela 1).

Neste estudo as ISTs mais prevalentes foram Sífilis (44\%), HPV (43\%) e cervicite (11\%). Além disso, 16\% das mulheres estudadas apresentavam duas ISTs concomitantemente, em linha com estudos que associam a presença de uma IST como fator 
de risco para outras (Aguirrebengoa et al, 2020). Destaca-se que a vaginose, embora não seja considerada IST, foi estudada neste trabalho devido a sua relevância como fator de risco para desenvolvimento de outras ISTs (Ministério da Saúde, 2020).

Tabela 1 - Dados coletados de mulheres com Infecções Sexualmente Transmissíveis (ISTs) em Cascavel-PR no ano de $2019(\mathrm{n}=127)$.

\begin{tabular}{|c|c|c|}
\hline Escolaridade da mãe & Mulheres & Porcentagem \\
\hline Ensino Fundamental Incompleto & 3 & $2 \%$ \\
\hline Ensino Fundamental Completo & 12 & $10 \%$ \\
\hline Ensino Médio Incompleto & 11 & $9 \%$ \\
\hline Ensino Médio Completo & 65 & $51 \%$ \\
\hline Ensino Superior Incompleto & 13 & $10 \%$ \\
\hline Ensino Superior Completo & 23 & $18 \%$ \\
\hline \multicolumn{3}{|l|}{ Prevalência de ISTs } \\
\hline Sífilis & 56 & $44 \%$ \\
\hline HPV & 43 & $34 \%$ \\
\hline Vaginose & 16 & $13 \%$ \\
\hline Cervicite & 14 & $11 \%$ \\
\hline Herpes Simples & 11 & $9 \%$ \\
\hline HTLV & 1 & $1 \%$ \\
\hline Doença Inflamatória Pélvica & 4 & $3 \%$ \\
\hline Síndrome do Corrimento Ureteral & 1 & $1 \%$ \\
\hline Molusco Contagioso & 3 & $2 \%$ \\
\hline Mais que uma IST & 20 & $16 \%$ \\
\hline \multicolumn{3}{|l|}{ Método Contraceptivo } \\
\hline $\begin{array}{l}\text { Apenas anticoncepcional hormonal } \\
\text { oral ou dispositivo intrauterino (DIU) }\end{array}$ & 41 & $32 \%$ \\
\hline Anticoncepcional hormonal oral ou & 34 & $26 \%$ \\
\hline \multicolumn{3}{|l|}{ DIU associado com preservativo } \\
\hline Apenas preservativo & 12 & $9 \%$ \\
\hline Coito Interrompido & 2 & $2 \%$ \\
\hline Abstinência & 2 & $2 \%$ \\
\hline Laqueadura & 1 & $1 \%$ \\
\hline Não informado & 18 & $14 \%$ \\
\hline Não usam método contraceptivo & 17 & $14 \%$ \\
\hline \multicolumn{3}{|c|}{ Quantidade de parceiros sexuais nos últimos 12 meses } \\
\hline Nenhum & 1 & $1 \%$ \\
\hline Um parceiro & 75 & $59 \%$ \\
\hline Dois parceiros & 17 & $14 \%$ \\
\hline Três a quatro parceiros & 18 & $14 \%$ \\
\hline Cinco a dez parceiros & 8 & $6 \%$ \\
\hline Mais que dez parceiros & 3 & $2 \%$ \\
\hline Não informado & 5 & $4 \%$ \\
\hline \multicolumn{3}{|l|}{ Tabagista } \\
\hline Sim & 11 & $9 \%$ \\
\hline Não & 97 & $76 \%$ \\
\hline Não informado & 19 & $15 \%$ \\
\hline \multicolumn{3}{|l|}{ Orientaç̃o Sexual } \\
\hline Heterossexual & 123 & $97 \%$ \\
\hline Bissexual & 4 & $3 \%$ \\
\hline
\end{tabular}

Fonte: Autores (2021).

Cerca de $36 \%$ das mulheres relataram que tiveram relações sexuais com duas ou mais parcerias sexuais nos últimos 12 meses. Considerando que maior quantidade de parceiros sexuais é fator de risco para desenvolvimento de ISTs (Jung, 2019) é importante que elas utilizem métodos contraceptivos de barreira para prevenir desenvolvimento de ISTs. Na análise dos dados foi possível associar a maior prevalência de uso de preservativo de barreira em mulheres com dois ou mais parceiros nos últimos 12 meses ( $\mathrm{p}=0,013)$ (Jung, 2019). 
Como esse projeto estuda apenas mulheres diagnosticadas com ISTs atendidas em um centro de referência em atenção secundária, foi necessário avaliar também as práticas contraceptivas de mulheres no geral para complementar a análise, utilizando como base o artigo de Lago et al. (2020), intitulado "Diferenciais da prática contraceptiva no Município de São Paulo, Brasil: resultados do inquérito populacional Ouvindo Mulheres". Assumindo que a amostra de mulheres do artigo seja equivalente à população feminina de Cascavel - PR, os percentuais de métodos contraceptivos escolhidos foram analisados e comparados entre os dois grupos.

Então, através de análises inferenciais dos dados obtidos no estudo e de informações descritas no artigo de Lago et al (2020) foi possível estabelecer hipóteses com valor estatisticamente significativo, ou seja, valor $p<0,05$. Juntamente com as hipóteses relevantes foram calculados a razão de chances e os intervalos de confiança de 95\%. Como exposto na Tabela 2, uma dessas hipóteses é que mulheres que utilizam apenas contraceptivo de barreira contraem menos ISTs do que mulheres que escolhem outros métodos contraceptivos, como é o caso dos anticoncepcionais orais $(\mathrm{p}=0,003)$.

Tabela 2 - Correlações estatisticamente significativas.

\begin{tabular}{lcc}
\hline Descrição da hipótese & Valor p & $\begin{array}{c}\text { Razão de Chances } \\
\text { (IC 95\%) }\end{array}$ \\
\hline $\begin{array}{l}\text { Mulheres com ISTs têm menor prevalência na utilização de apenas } \\
\text { método de barreira como método contraceptivo }\end{array}$ & 0.003 & 0,41 \\
$\begin{array}{l}\text { Maior prevalência de uso de preservativo de barreira (associado ou não } \\
\text { com método hormonal) como método contraceptivo em mulheres com } \\
\text { dois ou mais parceiros sexuais nos últimos 12 meses }\end{array}$ & 0.013 & 2.65 \\
\hline
\end{tabular}

Fonte: Autores (2021).

\section{Considerações Finais}

Embora haja uma grande variedade de métodos contraceptivos capazes de prevenir uma gravidez indesejada e os anticoncepcionais hormonais sejam muito eficazes para esse propósito, impedir a transmissão de ISTs é possível apenas através dos preservativos de barreira masculino e feminino. Sendo assim, através de uma análise transversal, averiguou-se a influência dos métodos contraceptivos hormonais na disseminação de ISTs, analisando prontuários de pacientes do CEDIP.

Mulheres com ISTs apresentam menor prevalência de utilização de preservativo de barreira como método contraceptivo, na comparação com mulheres sem ISTs (Tabela 2, p=0,003). Uma das hipóteses para este fenômeno é que a segurança passada pelos anticoncepcionais na prevenção da gravidez pode reduzir a utilização de métodos contraceptivos de barreira, que previnem ISTs. Dessa forma, é essencial que profissionais de saúde informem mulheres sobre os benefícios da associação de métodos de barreira na proteção contra ISTs.

Houve associação estatisticamente relevante de mulheres que relataram duas ou mais parcerias sexuais nos últimos 12 meses com maior prevalência na utilização de métodos contraceptivos de barreira $(\mathrm{p}=0,013)$ (Tabela 2). Considerando que elas apresentam fator de risco para desenvolvimento de ISTs, os profissionais de saúde devem recomendar a utilização de métodos contraceptivos de barreira em todas as relações sexuais para haver maior proteção contra desenvolvimento de ISTs.

Portanto, para haver máxima proteção contra ISTs e de gravidez, sugere-se a utilização combinada de anticoncepcional hormonal ou DIU associado com método de barreira, como o preservativo masculino, especialmente em mulheres com maior risco de desenvolvimento de ISTs, como adolescentes e mulheres com várias parcerias sexuais e, idealmente, em todas as mulheres com atividade sexual. 
Futuros estudos podem abordar a temática de ISTs em mulheres jovens ao estudar uma amostra maior e também incluir participantes sem diagnóstico de ISTs para avaliar os fatores que estão mais associados ao risco de desenvolvimento dessas patologias nesta população.

\section{Referências}

Aguirrebengoa, O. A., Garcia, M. V., Sanchez, M. R., D’Elia, G., Méndez, B. C., Arrancudiaga, M. A., León, S. B., López, T. P., Escribano, P. C., Martín, J. B., Prieto, B. M., Fuentes, M. E., Lotero, M. G., Utrilla, M. R., Martín, C. R., \& Del Romero Guerrero, J. (2020). Risk factors associated with sexually transmitted infections and HIV among adolescents in a reference clinic in Madrid. PLOS ONE, 15(3), 1-10. https://doi.org/10.1371/journal.pone.0228998

Apter, D. (2018). Contraception options: Aspects unique to adolescent and young adult. Best Practice and Research: Clinical Obstetrics and Gynaecology, 48(October), 115-127. https://doi.org/10.1016/j.bpobgyn.2017.09.010

Baeten, J. M., Nyange, P. M., Richardson, B. A., Lavreys, L., Chohan, B., Martin, H. L., Mandaliya, K., Ndinya-Achola, J. O., Bwayo, J. J., \& Kreiss, J. K. (2001). Hormonal contraception and risk of sexually transmitted disease acquisition: Results from a prospective study. American Journal of Obstetrics and Gynecology, 185(2), 380-385. https://doi.org/10.1067/mob.2001.115862

Baldivia, G. C., \& Graudenz, G. S. (2015). Typical case: Herpes zoster. Revista Da Associacao Medica Brasileira, 61(2), 114-115. https://doi.org/10.1590/18069282.61.02.114

Brasil. (2015). Biblioteca Virtual em Saúde.

Centers For Disease Control and Prevention. (2018). Sexually Transmitted Disease Surveillance 2018.

Centers For Disease Control and Prevention. (2015). Sexually Transmitted Diseases Treatment Guidelines, 2015 (Vol. 64). https://doi.org/10.1097/00008480200308000-00006

Chan, P. A., Robinette, A., Montgomery, M., Almonte, A., Cu-Uvin, S., Lonks, J. R., Chapin, K. C., Kojic, E. M., \& Hardy, E. J. (2016). Extragenital Infections Caused by Chlamydia trachomatis and Neisseria gonorrhoeae: A Review of the Literature. Infectious Diseases in Obstetrics and Gynecology, 2016. https://doi.org/10.1155/2016/5758387

Colquitt, C. W., \& Martin, T. S. (2017). Contraceptive Methods: A Review of Nonbarrier and Barrier Products. Journal of Pharmacy Practice, 30(1), 130-135. https://doi.org/10.1177/0897190015585751

Crosbie, E. J., Einstein, M. H., Franceschi, S., \& Kitchener, H. C. (2013). Human papillomavirus and cervical cancer. The Lancet, 382(9895), 889-899. https://doi.org/10.1016/S0140-6736(13)60022-7

De Martel, C., Ferlay, J., Franceschi, S., Vignat, J., Bray, F., Forman, D., \& Plummer, M. (2012). Global burden of cancers attributable to infections in 2008: A review and synthetic analysis. The Lancet Oncology, 13(6), 607-615. https://doi.org/10.1016/S1470-2045(12)70137-7

Decker, C. F. (2016). Sexually transmitted diseases: An overview. Disease-a-Month, 62(8), 258-259. https://doi.org/10.1016/j.disamonth.2016.03.008

Den Heijer, C. D. J., Hoebe, C. J. P. A., Driessen, J. H. M., Wolffs, P., Van Den Broek, I. V. F., Hoenderboom, B. M., Williams, R., De Vries, F., \& DukersMuijrers, N. H. T. M. (2019). Chlamydia trachomatis and the Risk of Pelvic Inflammatory Disease, Ectopic Pregnancy, and Female Infertility: A Retrospective Cohort Study among Primary Care Patients. Clinical Infectious Diseases, 69(9), 1517-1525. https://doi.org/10.1093/cid/ciz429

Dos Santos, M. M., Lopes, A. K. B., Roncalli, A. G., \& De Lima, K. C. (2020). Trends of syphilis in Brazil: A growth portrait of the treponemic epidemic. PLoS ONE, 15(4), 1-11. https://doi.org/10.1371/journal.pone.0231029

Fuchs, W., \& Brockmeyer, N. H. (2014). Sexually Transmitted Infections. Deutsche Dermatologische Gesellschaft, 12, 451-464. https://doi.org/10.1016/j.emc.2019.07.009

Gallo, M. F., Grimes, D. A., Lopez, L. M., \& Schulz, K. F. (2013). Nonlatex versus latex male condoms for contraception. Cochrane Database of Systematic Reviews, 1. https://doi.org/10.1002/14651858.cd003550.pub2

Galvin, S. R., \& Cohen, M. S. (2004). The role of sexually transmitted diseases in HIV transmission. Nature Reviews Microbiology, 2(1), 33-42. https://doi.org/10.1038/nrmicro794

Grandi, G., Barra, F., Ferrero, S., Sileo, F. G., Bertucci, E., Napolitano, A., \& Facchinetti, F. (2019). Hormonal contraception in women with endometriosis: a systematic review. European Journal of Contraception and Reproductive Health Care, 24(1), 61-70. https://doi.org/10.1080/13625187.2018.1550576

Holmes, K. K., Levine, R., \& Weaver, M. (2004). Effectiveness of condoms in preventing sexually transmitted infections. Bulletin of the World Health Organization, 82(6), 454-461. https://doi.org/10.1590/S0042-96862004000600012

Jung, M. (2019). Risk factors of sexually transmitted infections among female sex workers in Republic of Korea. Infectious Diseases of Poverty, 8(1), 1-8. https://doi.org/10.1186/s40249-019-0516-x

Kissinger, P., \& Adamski, A. (2013). Trichomoniasis and HIV interactions: A review. Sexually Transmitted Infections, 89(6), 426-433. https://doi.org/10.1136/sextrans-2012-051005

Lago, T. D. G. do, Kalckmann, S., Alves, M. C. G. P., Escuder, M. M. L., Koyama, M., \& Barbosa, R. M. (2020). Diferenciais da prática contraceptiva no Município de São Paulo, Brasil: resultados do inquérito populacional Ouvindo Mulheres. Cadernos de Saúde Pública, 36(10). https://doi.org/10.1590/0102$311 \times 00096919$ 
Research, Society and Development, v. 10, n. 10, e124101018642, 2021

(CC BY 4.0) | ISSN 2525-3409 | DOI: http://dx.doi.org/10.33448/rsd-v10i10.18642

Mansour, D., Inki, P., \& Gemzell-Danielsson, K. (2010). Efficacy of contraceptive methods: A review of the literature. European Journal of Contraception and Reproductive Health Care, 15(1), 4-16. https://doi.org/10.3109/13625180903427675

McCormack, D., \& Koons, K. (2019). Sexually Transmitted Infections. Emergency Medicine Clinics of North America, 37(4), 725-738. https://doi.org/10.1016/j.emc.2019.07.009

Ministério da Saúde. (2020). Protocolo Clínico e Diretrizes Terapêuticas para Atenção Integral às Pessoas com Infecções Sexualmente Transmissíveis (IST).

Mishori, R., McClaskey, E. L., \& Winklerprins, V. J. (2012). Chlamydia Trachomatis infections: Screening, diagnosis, and management. American Family Physician, 86(12), 1127-1132.

Mitchell, C., \& Prabhu, M. (2013). Pelvic inflammatory disease: Current concepts in pathogenesis, diagnosis and treatment. Infectious Disease Clinics of North America, 27(4), 793-809. https://doi.org/10.1016/j.idc.2013.08.004

Organização Mundial da Saúde. (2019). Sexually transmitted infections (STIs).

Park, S. T., Lee, S. W., Kim, M. J., Kang, Y. M., Moon, H. M., \& Rhim, C. C. (2017). Clinical characteristics of genital chlamydia infection in pelvic inflammatory disease. BMC Women's Health, 17(1), 1-7. https://doi.org/10.1186/s12905-016-0356-9

Pereira A. S. et al. (2018). Metodologia da pesquisa científica. UFSM.

Petry, S., Padilha, M. I., Eich Kuhnen, A., \& Horner Schlindwein Meirelles, B. (2019). Saberes de estudantes de enfermagem sobre a prevenção de Infecções Sexualmente Transmissíveis. Revista Brasileira de Enfermagem, 72(5), 1208-1216. http://search.ebscohost.com/login.aspx?direct=true\&db= rzh\&AN=138820234\&site=ehost-live

Raidoo, S., \& Kaneshiro, B. (2017). Contraception counseling for adolescents. Current Opinion in Obstetrics and Gynecology, 29(5), 310-315. https://doi.org/10.1097/GCO.0000000000000390

Rouzine, I. M. (2020). An evolutionary model of progression to aids. Microorganisms, 8(11), 1-15. https://doi.org/10.3390/microorganisms8111714

Sanjosé, S., Díaz, M., Castellsagué, X., Clifford, G., \& Bruni, L. (2007). Worldwide prevalence and genotype distribution of cervical HPV in women with normal cytology. Lancet Infect, 7(7), 453-459. http://www.ncbi.nlm.nih.gov/pubmed/17597569

Serfaty, D. (2019). Update on the contraceptive contraindications. Journal of Gynecology Obstetrics and Human Reproduction, 48(5), $297-307$. https://doi.org/10.1016/j.jogoh.2019.02.006

Vieira, E. M., Badiani, R., Dal Fabbro, A. L., \& Rodrigues Junior, A. L. (2002). Características do uso de métodos anticoncepcionais no Estado de São Paulo. Revista de Saúde Pública, 36(3), 263-270. https://doi.org/10.1590/s0034-89102002000300002

Wagenlehner, F. M. E., Brockmeyer, N. H., Discher, T., Friese, K., \& Wichelhaus, T. A. (2016). Klinik, Diagnostik und Therapie sexuell öbertragbarer Infektionen. Deutsches Arzteblatt International, 113(1-2), 11-22. https://doi.org/10.3238/arztebl.2016.0011 